$X V^{e ̀ m e s}$ Journées Nationales Génie Côtier - Génie Civil

La Rochelle, 29 au 31 mai 2018

DOI:10.5150/jngcgc.2018.035 @ Editions Paralia CFL

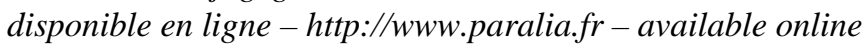

\title{
Caractéristiques du climat de houle au large de la côte ivoirienne entre 1985 et 2015 : impacts sur l'évolution morpho dynamique du rivage
}

\author{
Kouadio Salomon YAO ${ }^{1}$, Nadi Paul DANGUI ${ }^{3}$, Male GBEGBE ${ }^{2}$, Jacques ABE ${ }^{1}$ \\ 1. Laboratoire de Physique et de Géologie Marine, Centre de Recherches \\ Océanologiques-Abidjan.29 rue des Pêcheurs, Treichville, 01 BP V 18 Abidjan, \\ Côte d'Ivoire.yksalomon3@yahoo.fr ; jacquesabe@yahoo.com
}

2. Laboratoire des Géosciences Marines, UFR Sciences de la Terre et des Ressources Minières, Université Félix Houphouët Boigny- Abidjan, Côte d'Ivoire. malegbegbe@yahoo.fr

3. Laboratoire de traitement d'images et de l'information géographique (LATIG). Institut de Géographie Tropicale (IGT), Université Félix Houphouët Boigny- Abidjan, Côte d'Ivoire.danguinadi@yahoo.fr

\section{Résumé :}

La caractérisation à long terme (30 ans) des conditions de houle au large de la côte ivoirienne, a été effectuée grâce à l'analyse des données altimétriques issues des projets ERA-40 et ERA-Intérim. Les résultats révèlent que 99\% des hauteurs significatives des houles du littoral sont inférieures à $2 \mathrm{~m}$. Elles constituent le principal moteur de l'évolution morpho dynamique du littoral. Leur répartition annuelle permet de distinguer deux saisons de houles : une saison de houles énergiques de mai à septembre et une saison de houle faibles de septembre à avril. Ces houles proviennent principalement des directions Sud (53\%) et Sud-Sud-ouest (45\%). On note en outre une occurrence de houles très énergiques ou houles de tempêtes dont les fréquences d'occurrence sont susceptibles d'expliquer les modifications profondes du rivage (érosion considérable), observées sur le littoral au cours de la période 1985 - 2015.

Mots clés : Houle, Houle de tempête, Evolution morphodynamique, Littoral, Côte d'Ivoire.

\footnotetext{
Abstract:

The long-term (30 years) wave climate off the Ivorian coasts have been characterized using altimetry data analysis collected from ERA-40 and ERA-Interim projects. Results show that $99 \%$ of significant coastal waves' heights are less than $2 \mathrm{~m}$. They are the main parameters explaining the coastal morpho dynamic changes. Their annual distribution can be divided into two seasonal regimes: a season of high waves' energy spreading from May to September and a low waves' energy season from September to April. These waves are originating from South (53\%) and South-South-West (45\%).
} 


\section{Thème 2 - Dynamique sédimentaire}

The occurrence of very high waves' energy also called stormy waves, correspond to the highest morphological changes observed on the coast during the years 1985 to 2015 .

Keywords: Wave, Storm, Morphodynamic changes, Coastline, Côte d'Ivoire.

\section{Introduction}

Le littoral ivoirien est sujet à une érosion du rivage, préoccupante surtout sur la basse côte sableuse du bassin sédimentaire, en raison des enjeux multiformes dans cette zone. Les travaux de recherche y afférent mettent en évidence les tendances évolutives et les secteurs les plus sensibles (HAUHOUOT, 2000 ; AFFIAN et al., 2003 ; ABE, 2005 ; YAO et al., 2010 ; TOURE et al., 2012 ; KONAN et al., 2009 \& 2014 ; KONAN, 2012 ; N'DOUFFOU, 2012 ; YAO, 2012). Certains travaux révèlent que les profondes modifications morphologiques du rivage et les forts taux de recul sont consécutifs à l'occurrence des agitations exceptionnelles, souvent assimilées à des houles de tempêtes. Toutefois, il convient de noter que ces études abordent rarement l'analyse conjointe de la spécification des caractéristiques des houles de tempête et des impacts observés sur le littoral, au cours des périodes ayant enregistré des perturbations morphologiques considérables. La présente communication se propose donc de caractériser les états de mer au large de la côte ivoirienne et de les mettre en relation avec les grands épisodes érosifs observés en Côte d'Ivoire.

\section{Présentation de la zone d'étude et méthodologie}

\subsection{Zone d'étude}

Le littoral ivoirien est situé en Afrique de l'Ouest, dans le golfe de Guinée, entre les longitudes $2^{\circ} 25^{\prime}$ et $7^{\circ} 30^{\prime} \mathrm{W}$ et les latitudes $4^{\circ} 00^{\prime}$ et $5^{\circ} 30^{\prime} \mathrm{N}$. Il s'étire sur environ 600 km, du Cap des Palmes (Liberia) à l'Ouest au Cap des Trois Pointes (Ghana) à l'Est. Cette zone est caractérisée d'Ouest en Est par une variation lithologique des formations géologiques en contact avec l'océan, définissant ainsi deux types de côte géomorphologiquement distincts (figure 1). La zone de socle à l'Ouest (de Tabou à Sassandra), enregistre une côte de plateaux (côte de falaises vives ou mortes) en échelon où les segments de plages alternent avec des promontoires rocheux constitués de formations précambriennes. De Sassandra à l'extrême Est du littoral, les formations sédimentaires constituées de sables argileux du continental terminal (mio-pliocène) forment des falaises vives dans les environs de Fresco. De l'Est de la passe de Fresco à Assinie, ces formations sont relayées par les bas cordons sableux quaternaires de faible à forte extension qui isolent de l'océan les systèmes lagunaires. Cette zone présente un tracé quasi rectiligne de la côte avec un point d'inflexion au droit du Canyon sous-marin "le Trou Sans Fond" à Abidjan. 


\section{XVèmes Journées Nationales Génie Côtier - Génie Civil \\ La Rochelle, 29 au 31 mai 2018}

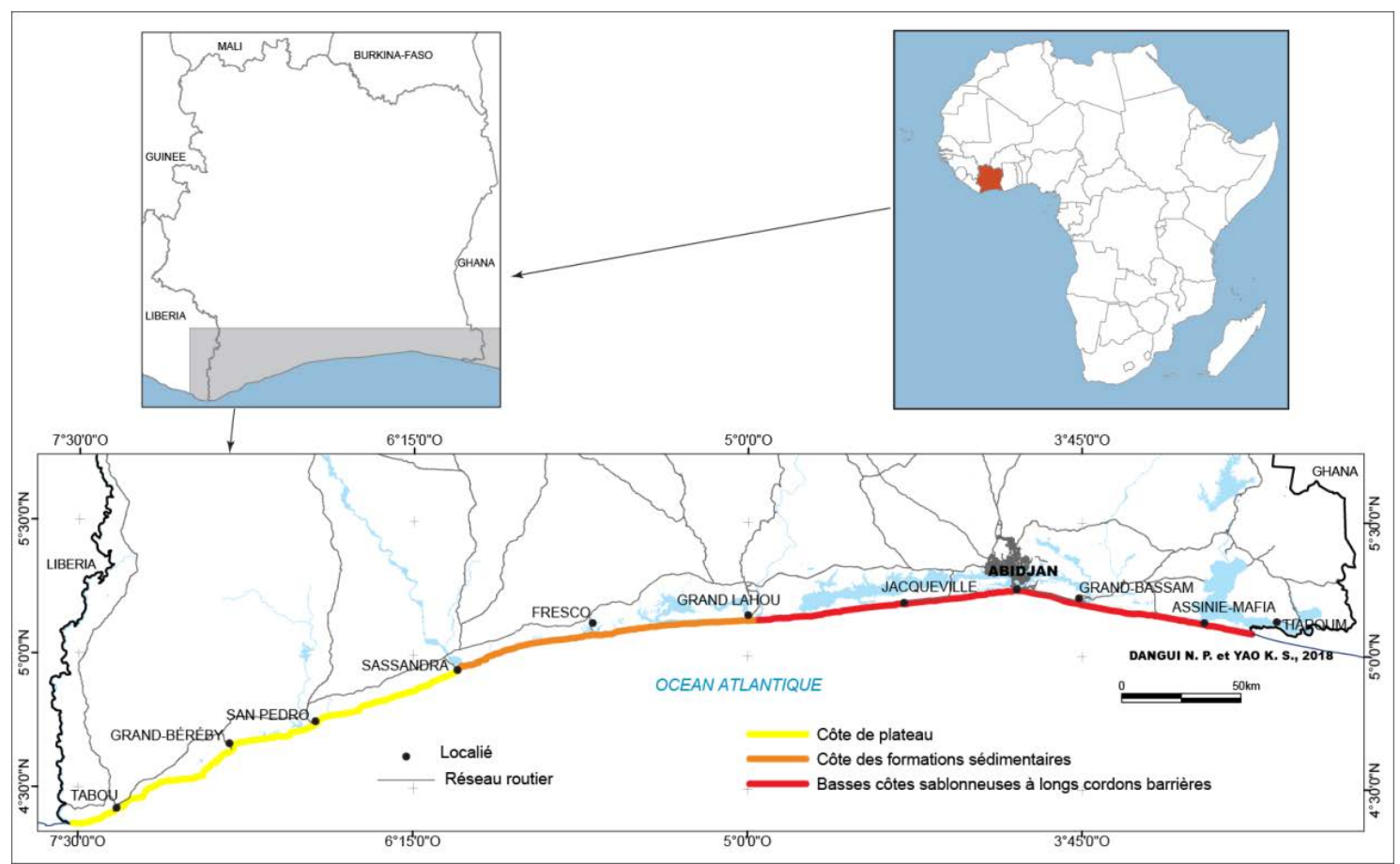

Figure 1.Carte géomorphologique du littoral ivoirien.

\subsection{Méthodologie}

Les états de mer sont généralement caractérisés par la Hauteur significative $(H s)$ des houles. Dans cette étude, les données utilisées sont issues des projets ERA-40 et ERAIntérim. Celles retenues pour analyse s'étalent sur 30 années d'observation, de 1985 à 2015. Ces données ont été validées par comparaison aux données des satellites océanographiques AVISO (www.Aviso.oceanobs.com), le long du littoral ivoirien (TOUALY et al., 2015). Le choix des données altimétriques AVISO comme référence, notamment la Hauteur significative des houles (Hs) réside dans le fait qu'elles ont été validées par comparaisons aux mesures in situ, dans l'hémisphère Nord, autour des côtes de l'Amérique du Nord et en Europe (ABDALLA et al., 2008).Les points d'observation sont suffisamment situés au large de la côte sur fond de $20 \mathrm{~m}$, où la houle ne semble pas encore être soumise aux effets locaux (gonflement et réfraction). Outre les Hauteurs significatives $(H s)$, les périodes de pic $(T p)$ et les directions de pic $(D p)$ des houles ont été également analysées. Elles ont été exportées à partir du site: http://www.ecmwf.int. Le logiciel MATLAB a permis de les filtrer et moyenner sur la surface comprise entre les longitudes $7^{\circ} 30^{\prime}$ et $2^{\circ} 25^{\prime} \mathrm{W}$ et les latitudes $4^{\circ}$ et $6^{\circ} \mathrm{N}$, afin d'obtenir des données assimilables (série chronologique quadri journalière avec un pas de temps de 6 heures). Trois types d'analyse ont été réalisés pour mettre en évidence l'évolution des hauteurs significatives, des périodes de pic et des directions de pic des houles. Elles concernent:

- l'analyse statistique classique; 


\section{Thème 2 - Dynamique sédimentaire}

- 1'analyse de la variabilité saisonnière annuelle des types de houles et;

- l'analyse de l'occurrence des houles de tempête. Cette dernière analyse permet de faire la corrélation entre les enregistrements altimétriques et les impacts des agitations exceptionnelles observées sur le littoral ivoirien.

\section{Résultats et discussions}

3.1 Caractéristiques des houles sur le littoral ivoirien entre 1985 et 2015

L'analyse statistique des caractéristiques des houles entre 1985 et 2015, montre que les hauteurs significatives $(\mathrm{Hs})$ sont comprises entre $0,50 \mathrm{~m}$ et $2,80 \mathrm{~m}$. Elles sont majoritairement inférieures à $2 \mathrm{~m}$ (99\%). La moyenne observée est de 1,24 m. Si l'on se réfère à la classification des houles selon TASTET et al. (1985), les houles faibles $(H s(m)<1)$ représentent $20 \%$ des observations. Les houles moyennes $(1<H s(m)<1,80)$ et les houles fortes $(1,8<H s(m)<2)$ représentent respectivement $60 \%$ et $20 \%$.

Les périodes de pic (Tp) des houles oscillent entre 5 et $16 \mathrm{~s}$ avec une moyenne de $9,05 \mathrm{~s}$. Près de $60 \%$ des périodes de pic sont comprises entre 8 et $10 \mathrm{~s}$. Les houles de périodes inférieures à $8 \mathrm{~s}$ et comprise entre 10 et $12 \mathrm{~s}$ ont respectivement des fréquences d'occurrence de 20 et 19\%. Les houles de grandes longueurs d'onde ( $T p>12 \mathrm{~s}$ ) affichent une faible occurrence $(1 \%)$. Ces dernières enregistrent généralement les plus fortes hauteurs significatives $(H s(m)>2)$. Elles seraient à l'origine des modifications morphologiques profondes du littoral. Ces houles proviennent essentiellement des directions Sud et Sud-Sud-ouest (figure 2) comme le stipulent les travaux de TASTET et al. (1985). Les taux de prédominance de ces différentes directions sont respectivement de $53 \%$ et $45 \%$. Toutefois, il est à noter la présence d'une faible proportion de houles de direction Sud-Sud-est, avec un taux de 2\%.

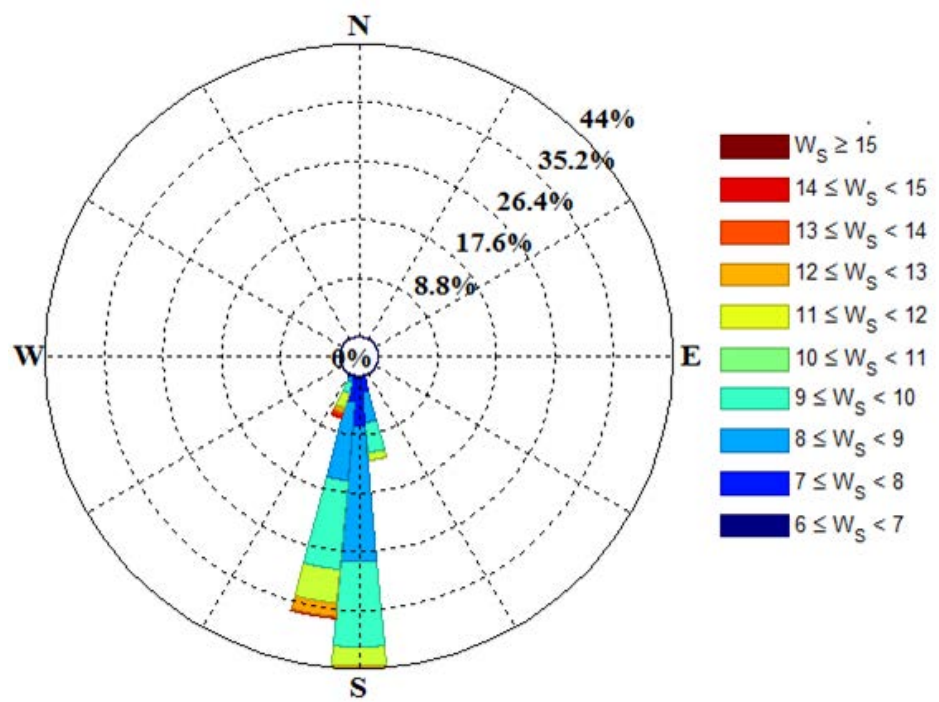

Figure 2.Rose des vagues au large de la Côte d'Ivoire. 


\section{XVèmes Journées Nationales Génie Côtier - Génie Civil \\ La Rochelle, 29 au 31 mai 2018}

\subsection{Répartition annuelle des houles sur le littoral ivoirien}

La répartition annuelle des différentes houles révèle une variation saisonnière du climat de houles (figure 3). Les houles énergiques ou fortes $(H s(m)>1,8)$ s'observent de mai à septembre. Les fréquences d'occurrences mensuelles de ces houles sont inférieures à $10 \%$. Cette période coïncide avec celle où la mousson ouest africaine atteint son maximum, avec des houles bien formées du fait de l'importance du fetch. Les houles moyennes $(1,0<H s(m)<1,8)$, s'observent toute l'année. Elles présentent une forte occurrence d'avril à novembre, avec une fréquence de 80 à $95 \%$. Cette phase se situe entre le recul de l'alizé continental et le début de la mousson. Les houles faibles $(H<1 \mathrm{~m})$ couvrent les mois de septembre à avril. Les fréquences d'occurrence mensuelle varient entre $5 \%$ et $50 \%$. Elles sont quasiment absentes de mai à août et ont des occurrences plus marquées pendant la saison sèche de décembre à mars.

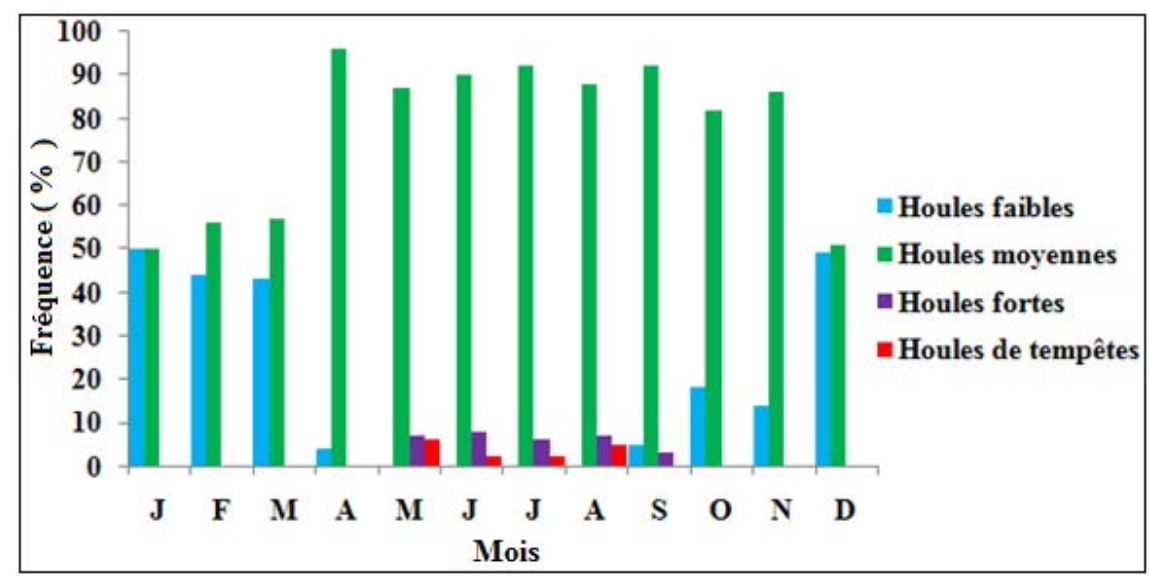

Figure 3. Distribution annuelle des différentes houles au large de la côte ivoirienne.

3.3 Occurrence des houles de tempête sur le littoral ivoirien de 1985 à 2015 et dynamique de la côte.

Les houles de tempête présentent des hauteurs significatives moyennes comprises entre 2,10 et 2,48 m. Les périodes de pic oscillent entre 9 et $14 \mathrm{~s}$. Ces houles proviennent généralement des directions Sud (52\%), Sud-Sud-ouest (45\%) et Sud-Sud-est (3\%). La répartition annuelle de ces houles indique leur occurrence de mai à septembre, avec un fort taux de mai à août (figure 3). Comme souligné précédemment, leur occurrence est donc en relation avec la période d'intensification de la mousson ouest africaine. Sur le littoral ivoirien, les événements tempétueux sont plus récurrents sur la période 19922015 avec une intensification de 1992 à 2002, contrairement à la période 1985-1992 (figure 4). La côte connaît naturellement une évolution à un rythme saisonnier qui se caractérise par une alternance de périodes d'érosion (démaigrissement de la plage et recul du trait de côte) entre les mois de mai et août et d'engraissement (accumulation de la plage) entre novembre et mars voire avril. Cette évolution est perceptible avec les 


\section{Thème 2 - Dynamique sédimentaire}

variations morphologiques observées au niveau des profils de suivi de l'évolution de la côte (ABE, 2005 ; YAO, 2012). Ces changements morphologiques sont plus remarquables sous l'action des évènements météo-marins de type houle de tempête (KOFFI et al., 2014 ; KONAN et al., 2016). Ces dernières années, les effets des agitations de août 2007, août 2011, fin mai 2014 restent profondément marqués dans la mémoire collective des riverains sur le littoral. La figure 5 illustre sur la basse côte sableuse entre Grand Lahou et Assinie, notamment à Abidjan, Grand-Bassam et Assinie, l'ampleur de leurs effets (destruction de maisons et d'équipements). Les fortes agitations d'août 2011, ont imprimé au rivage un recul de l'ordre de 3 à $7 \mathrm{~m}$ par endroits.

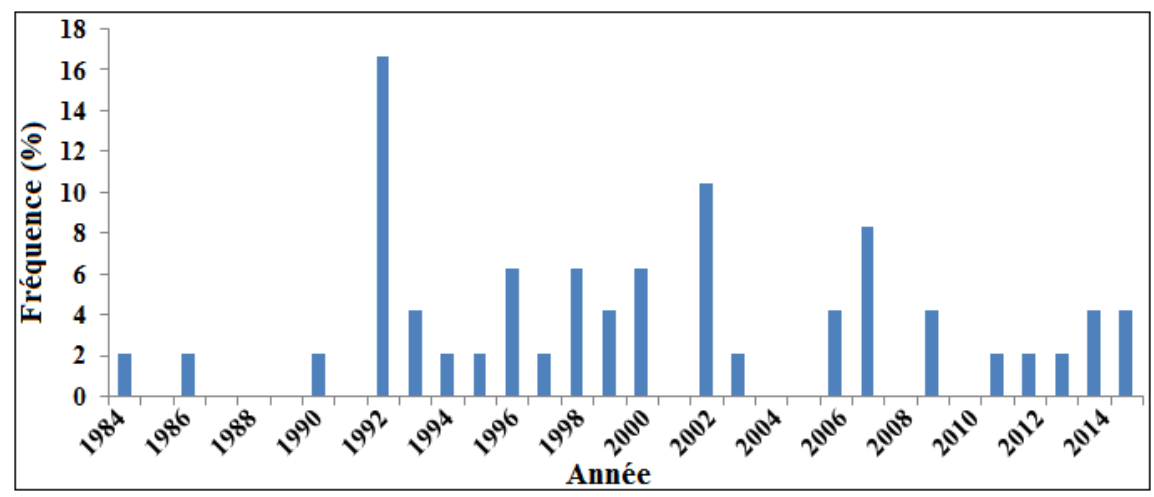

Figure 4. Fréquences d'occurrences annulles des houles de tempêtes sur le littoral ivoirien.

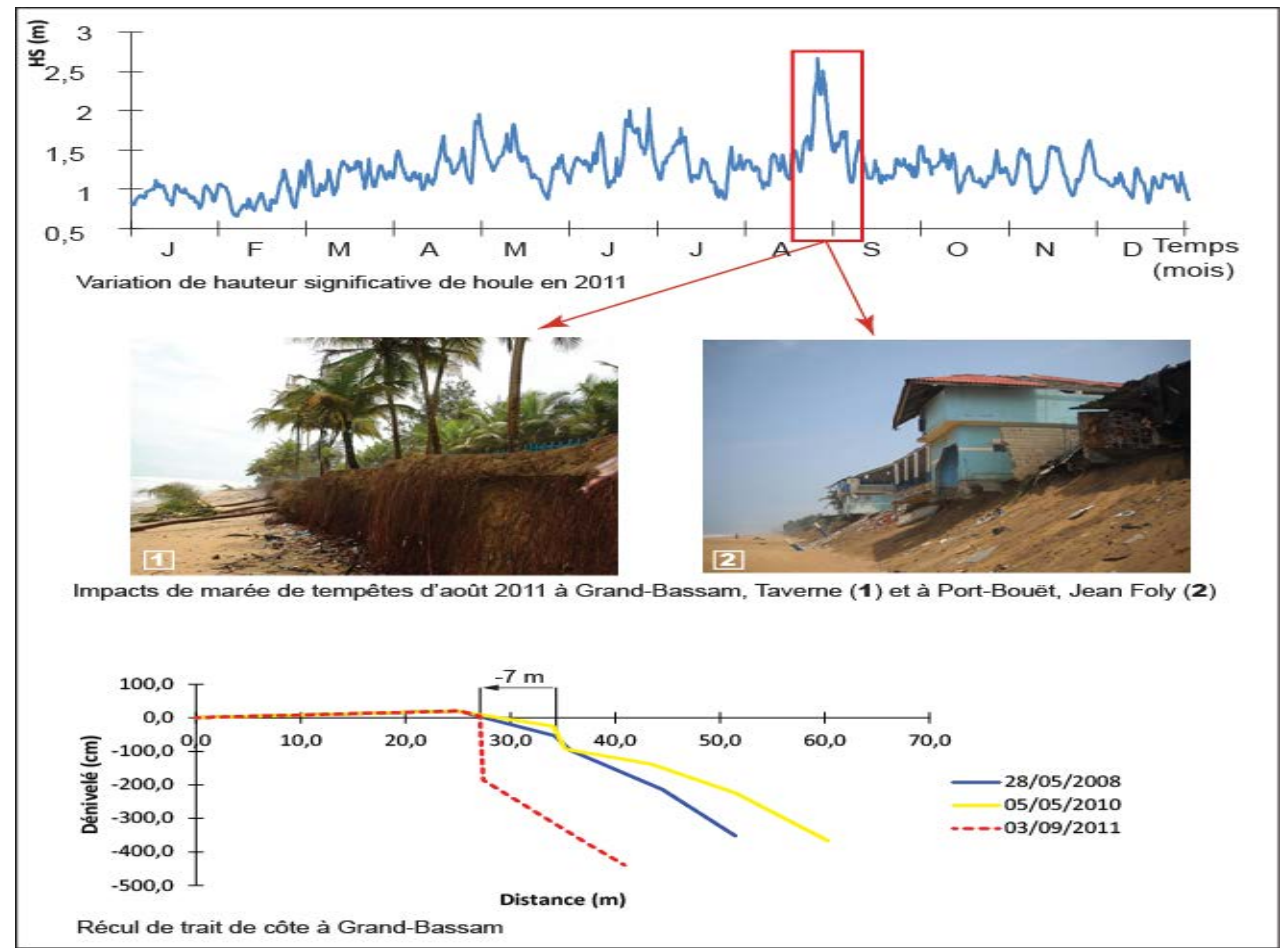

Figure 5. Impact de la houle de tempête d'août 2011 sur le littoral de Port Bouet et Grand Bassam. 


\section{XVèmes Journées Nationales Génie Côtier - Génie Civil \\ La Rochelle, 29 au 31 mai 2018}

\section{Conclusion}

Les houles au large de la côte ivoirienne présentent des hauteurs significatives moyennes $(\mathrm{Hs})$ comprises entre $0,50 \mathrm{~m}$ et $2,80 \mathrm{~m}$ et des périodes de 5 à $16 \mathrm{~s}$. Les houles de tempête ont des hauteurs significatives moyennes comprises entre 2,10 et 2,48 m avec des périodes qui oscillent entre 9 et $14 \mathrm{~s}$. Dans l'ensemble, les houles proviennent généralement des directions Sud et Sud-Sud-ouest. On note toutefois, une faible proportion provenant de la direction Sud-Sud-est. L'occurrence des climats de houle montre une variation saisonnière à l'exception des houles moyennes. Par ailleurs, l'analyse de l'occurrence des houles de tempêtes sur le littoral ivoirien entre 1985 et 2015 affiche deux grandes phases d'occurrence (1985-1992 et 1992-2015), avec une augmentation de leur fréquence entre 1992 et 2002. Ces houles sont le principal moteur de la dégradation profonde de la côte.

\section{Références bibliographiques}

ABDALLA S, JANSSEN P., BIDLOT J.R. (2008).Jason-2 OGDR wind and wave products: monitoring, validation and assimilation. Marine Geodesy, Vol. 33, Suppl. 1, pp 239-255. https://doi.org/10.1080/01490419.2010.487798

ABE J. (2005). Contribution à la connaissance de la morphologie et de la dynamique du littoral ivoirien (cas du littoral d'Abidjan) Essais de modélisation en vue d'une gestion rationnelle. Thèse, Université de Cocody-Abidjan, $345 \mathrm{p}$.

AFFIAN K., DIGBEHI Z., DJAGOUA E.M., KOUAME K.F., OUATTARA A. (2003).Utilisation des techniques de photographie aérienne et de profil de plage pour le contrôle de l'érosion côtière sur les segments de côte à Abidjan et à Grand-Bassam. Bioterre, Vol. 1(3), pp 53-67.

HAUHOUOT C. (2000).Analyse et cartographie de la dynamique du littoral et des risques naturels côtiers en Côte d'Ivoire. Thèse de Doctorat, Université de Nantes, $289 \mathrm{p}$.

KOFFI K. P.H., HAUHOUOT C., YAO K.S., DANGUI N.P., MONDE S., AKA K. (2014).Evolution à long terme (quarante dernières années) du trait de côte du périmètre littoral est de Port-Bouët et projection d'une ligne de recul. Revue de Géographie Tropicale et d'Environnement, $\mathrm{n}^{\circ}$ 2, pp 20-27

KONAN K.E., BAMBA S.-B., ABE J., AKA K. (2009). Impact des tempêtes récurrentes sur le modèle du périmètre littoral de Vridi-Port Bouët (Abidjan-Côte d'Ivoire). European Journal of Scientific Research, Vol. 28, pp 186-192.

KONAN K.E. (2012).Etude morpho-dynamique et sensibilité aux événements "exceptionnels" du cordon littoral sableux ivoirien à l'Est d'Abidjan (AbidjanAforenou). Thèse de Doctorat, Université Félix Houphouët Boigny d'Abidjan, 206 p.

KONANK E, N'GUESSAN Y.A., DJAGOUA E.M.V., AFFIAN K. (2014).Influence des houles exceptionnelles sur un cordon littoral étroit ivoirien à Azzureti. Geo-EcoTrop, Vol. 38 (1), pp 179-186. 


\section{Thème 2 - Dynamique sédimentaire}

KONAN E.K., ABE J., AKA K., NEUMEIER U., NYSSEN J., OZER A. (2016).Impacts des houles exceptionnelles sur le littoral ivoirien du Golfe de Guinée, Géomorphologie : relief, processus, environnement. Vol. 22(1), pp 105-120.

N'DOUFFOU G.H.C. (2012).Contribution morpho-sedimentologique et exoscopique à l'évolution du secteur du littoral ivoirien entre Sassandra et Abidjan. Thèse de Doctorat, Université Félix Houphouët Boigny d'Abidjan, 190 p.

TASTET J.P., CAILLON L., SIMON B. (1985). La dynamique sédimentaire littorale devant Abidjan. Impact des aménagements. Université nationale de Côte d'Ivoire; PAA, $39 \mathrm{p}$.

TOUALY E., AMAN A., KOFFI P., MARIN F., WANGO T.E. (2015). Ocean swell variability along the northern coast of the Gulf of Guinea. African Journal of Marine Science, Vol. 37(3), pp 353-361. https://doi.org/10.2989/1814232X.2015.1074940

TOURE B., KOUAME K.F., SOULEYE W., COLlET C., AFFIAN K., OZER A., RUDANT J.P., BIEMI J. (2012). L'influence des actions anthropiques dans l'évolution historique d'un littoral sableux à forte dérive sédimentaire: la baie de Port-Bouët (Abidjan, Côte d'Ivoire). Géomorphologie : relief, processus, environnement, Vol. 3, pp 113-126. https://doi.org/10.4000/geomorphologie. 9990

YAO K.S., ABE J., BAMBA S.B., KONAN K.E., AKA K. (2010).Dynamique d'un périmètre littoral portuaire : la côte de San-Pedro, sud-ouest de la Côte d'Ivoire. Rev. Paralia, Vol. 3, pp 2.1-2.12. https://doi.org/10.5150/revue-paralia.2010.002

YAO K.S. (2012). Etude de la dynamique sédimentaire du littoral occidental ivoirien entre Tabou et Sassandra: Approches morpho-bathymétriques, sédimentologiques et exoscopiques. Thèse de doctorat unique, université Félix Houphouët Boigny de Cocody-Abidjan, 197 p. 